\title{
Cambios en composición y abundancia de califóridos de interés forense en Bogotá
}

\author{
Misael López-Cepeda ${ }^{1,2}$, Giovanny Fagua ${ }^{3}$
}

\section{Change in composition and abundance of blowflies of forensic interest in Bogota}

\begin{abstract}
Calliphorids are the first arthropods to colonize cadavers, their composition and abundance vary according to location. They are used to estimate post-mortem intervals and surmise the relocation of corpses; making the identification of these local variations are key in detecting the relocation of a body. We assessed these blowfly variations in 11 sites in Bogotá. A pre-sampling (dry season) was conducted to select a trapping method and sampling sites; the formal sampling (rainy season) was conducted using a directed sweep net. We identified the following species: Lucilia sericata, Roraimomusca roraima, Compsomyiops verena, Calliphora nigribasis, Calliphora vicina and Sarconesiopsis magellanica and compared their richness, diversity, dominance and composition for each site. The highest richness was found in Chico-Lago (Chico) while the lowest was Alcazares (Barrios Unidos). Bolivia (Engativá) was the most diverse and 20 de Julio (San Cristobal) the least; dominance was higher in 20 de Julio and lower in Apogeo (Bosa). Species' composition and abundance varied between sites; this may be used as evidence to support cases in Bogotá in which cadavers have been relocated.
\end{abstract}

Keywords: Calliphoridae; composition and abundance; relocation of bodies; Bogotá.

Edited by Alberto Acosta四

1. Laboratorio de Entomología, Unidad de Ecología y Sistemática -UNESIS-, Departamento de Biología, Facultad de Ciencias, Pontificia

Universidad Javeriana, Bogotá D.C., Colombia.

2. Estudiante de maestría, Laboratorio de Genética Humana, Departamento de Biología, Facultad de Ciencias, Universidad de los Andes, Bogotá D.C., Colombia.

3. Laboratorio de Entomología, Grupo de Sistemática Molecular, Unidad de Ecología y Sistemática -UNESIS-, Departamento de Biología, Facultad de Ciencias, Pontificia Universidad Javeriana, Bogotá D.C., Colombia.

Received: 20-03-2014 Accepted: 25-05-2014

Published on line: 10-07-2014

Citation: López-Cepeda M, Fagua G (2015) Cambios en composición y abundancia de califóridos de interés forense en Bogotá. Universitas Scientiarum 20(1): 17-28 doi: 10.11144/Javeriana.SC20-1.ccac

Funding: Colciencias; Vicerrectoría de Investigaciones de la Pontificia Universidad Javeriana.

Electronic supplementary material: Supt. 1.

\section{Introducción}

La entomología médico-legal es la ciencia que analiza el uso de artrópodos como evidencia en investigaciones legales de muertes (Amendt et al. 2010). Su utilidad está basada en el hecho que los artrópodos colonizan cadáveres frescos rápidamente, utilizándolos como recurso alimenticio en forma secuencial y predecible haciendo de la descomposición cadavérica un proceso medible (Byrd \& Castner 2001). La aplicación más frecuentemente utilizada de la entomología médicolegal es la estimación del intervalo post-mortem (IPM), tiempo entre la muerte y el hallazgo del cadáver (Amendt et al. 2010). Su estimación depende en gran medida del conocimiento de la comunidad local de 
insectos de importancia forense y su abundancia en el área del crimen para la correcta interpretación de los datos (Fremdt \& Amendt 2013).

Los califóridos son considerados dentro de los insectos más importantes desde el punto de vista forense (Anderson 2000), pero su composición y abundancia puede variar según la época climática y hábitat (Brundage et al. 2011). Las preferencias de hábitat se asocian a mayor o menor abundancia de algunas especies en un sitio dado (Prinkkilä \& Hanski 1995). Algunas especies de califóridos son más frecuentes en ambientes naturales, otras lo son en ambientes transformados (Fremdt \& Amendt 2013) y cuando la abundancia es favorecida por las modificaciones de las condiciones medioambientales generadas por el hombre, se habla de sinantropía (Mariluis et al. 2008). Las ciudades son un mosaico que alterna ambientes completamente cubiertos por construcciones con ambientes con parques o zonas verdes de extensión variable, lo que genera gran variabilidad local en la composición de especies de cada sitio.

Bogotá, con 7’363.782 habitantes en 2010 (DANE 2005), fue la ciudad con más casos de suicidios y la segunda con más homicidios de Colombia durante 2011 (Macana 2012; Ricaurte 2012). En este contexto, el conocimiento de la variación en la composición y abundancia de califóridos puede ser de ayuda facilitando el uso de herramientas forenses entomológicas. Pinilla-Beltrán et al. (2012) establecieron el grado de sinantropía de la mayoría de especies de califóridos del área urbana de Bogotá basados en datos de un solo sitio urbano de muestreo. Ellos encontraron especies que se clasificaron como asinantrópicas, asociadas al ambiente forestal [Calliphora nigribasis (Macquart, 1851) y Roraimomusca roraima (Townsend, 1935)], hemisinantrópicas, asociadas al ambiente rural [Compsomyiops verena (Walker, 1849) y Sarconesiopsis magellanica (Le Guillou, 1842)] y sinantrópicas asociadas a ambientes urbanos [Calliphora vicina (Robineau-Desvoidy, 1851) y Lucilia sericata (Meigen, 1826)]. Sin embargo, Bogotá tiene ambientes urbanos con marcadas diferencias microclimáticas debido a la variación de las condiciones de altitud, temperatura (por efecto isla de calor), precipitación, brillo solar y nubosidad (IDEAM 2007). No se han estudiado las posibles variaciones locales en la composición y abundancia de califóridos dentro del área urbana de
Bogotá debido a las dificultades logísticas que esto implica. En este trabajo se identificaron variaciones en la composición y abundancia de califóridos en 11 ambientes urbanos (sitios) de diferentes localidades de Bogotá durante la época de lluvia.

\section{Materiales y métodos}

Área de estudio: Bogotá (4³6'43"N; 7404'07"W) se divide en 20 localidades. El área urbana abarca 19 de estas localidades y tiene una altitud promedio de $2630 \mathrm{~m}$ (Secretaria Distrital de Ambiente, SDA 2007). El área rural incluye la totalidad de la localidad de Sumapaz y parte del extremo oriental o sur de otras 10 localidades atravesando terrenos llanos y montañosos. Presenta un régimen pluviométrico bimodal, cuyos periodos más lluviosos son abril-mayo y septiembre-noviembre (IDEAM 2007). El área urbana de la ciudad presenta grandes diferencias en cuanto a las condiciones ambientales de sus localidades (SDA 2007).

Muestreo: Se realizó un pre-muestreo cualitativo y un muestreo cuantitativo formal. Durante el premuestreo se hizo una caracterización cualitativa de las zonas con mayor frecuencia de avistamientos de califóridos, dichas zonas fueron botaderos de basura, parques, rondas de rio y aceras con mayor presencia de residuos orgánicos (excremento humano y carne en descomposición). La caracterización cualitativa sirvió para definir zonas de captura en cada sitio para el muestreo cuantitativo formal. En el premuestreo también se evaluaron diferentes métodos de captura de califóridos: jameo con transecto, jameo con cebo (platos de diferentes colores con hígado en descomposición), jameo ad hoc direccionado (Villareal et al. 2006) y trampas descritas por FigueroaRoa \& Linhares (2002): contenedores oscuros perforados cebados con hígado en descomposición y con una bolsa como colector de insectos unida a la parte superior del contenedor. Dado que la mayor o menor presencia inmediata de desperdicios orgánicos en un sitio podía ser un factor alineado, se consideró ineficiente el uso de trampas, las cuales también podían ser destruidas por habitantes del sector. El pre-muestreo sirvió adicionalmente para obtener una colección de referencia e identificar las limitaciones logísticas de cada sitio potencial (vecindario, animales, seguridad, etc.). Se seleccionaron 11 sitios para 
el muestreo formal, definiendo al jameo ad hoc direccionado realizado siempre por el mismo colector como el método de colecta más conveniente debido a su alta especificidad, practicidad y eficiencia. Este consistió en la búsqueda activa de califóridos con jama siguiendo un recorrido circular predeterminado $(2 \mathrm{~km}$ aprox.) que pasa por los ambientes de congregación de califóridos del sitio (zonas con mayor frecuencia de avistamientos), volviendo al mismo punto de partida en un lapso de 90 minutos. Se usó una jama de red blanca.

El pre-muestreo se realizó durante la época seca (entre julio y septiembre de 2011), cuando se exploraron las siguientes 17 áreas incluidas en 9 localidades (Figura 1, Tabla 1): 1: Jardines del Recuerdo (Localidad de Suba); 2: Niza (Suba); 3: Humedal Córdoba (Suba); 4: Lisboa, Los Cedros y Santa Bárbara (Usaquén); 5: Bolivia (Engativá); 6: Garcés Navas (Engativá); 7: Alcázares (Barrios Unidos); 8: Parque Los Novios (Barrios Unidos); 9: Parque Simón Bolívar (Teusaquillo); 10: Chicó-Lago, Pardo Rubio y El Refugio (Chapinero); 11: campus Pontificia Universidad Javeriana y Parque Nacional Chapinero); 12: Alcalá (Puente Aranda); 13: Apogeo (Bosa); 14: Isla del Sol (Tunjuelito); 15: San José (Rafael Uribe Uribe); 16: 20 de Julio (San Cristóbal) y 17: Ciudad de Usme (Usme).

El muestreo formal (Figura 1) se realizó en la época de lluvias (16 a 30 de octubre de 2011), y los 11 sitios definidos fueron: 2. Niza (Suba), 4. Santa Bárbara, Los Cedros, Lisboa (Usaquén; en adelante Lisboa), 5. Bolivia (Engativá), 7. Alcázares (Barrios Unidos), 10. Chicó-Lago, Pardo Rubio y El Refugio (Chapinero; en adelante Chicó-Lago), 12. Alcalá (Puente Aranda), 13. Apogeo (Bosa), 14. Isla del Sol (Tunjuelito), 15. San José (Rafael Uribe Uribe), 16. 20 de julio (San Cristóbal) y 17. Ciudad de Usme (Usme). En cada sitio se evaluó la composición y abundancia de especies tomando cinco muestras. Las muestras fueron lo colectado en periodos de 90 minutos de duración durante los cuales se hacían colectas mediante jameo ad hoc direccionado. Las cinco muestras se tomaban de 8:00 a 9:30, de 9:30 a 11:00, de 11:00 a 12:30, de 12:30 a 14:00 y de 14:00 a 15:30 horas. Durante cada periodo se seguía un sendero definido para cada sitio de $2 \mathrm{~km}$ de longitud en promedio. Los califóridos capturados se sacrificaron en alcohol al 70\% y se

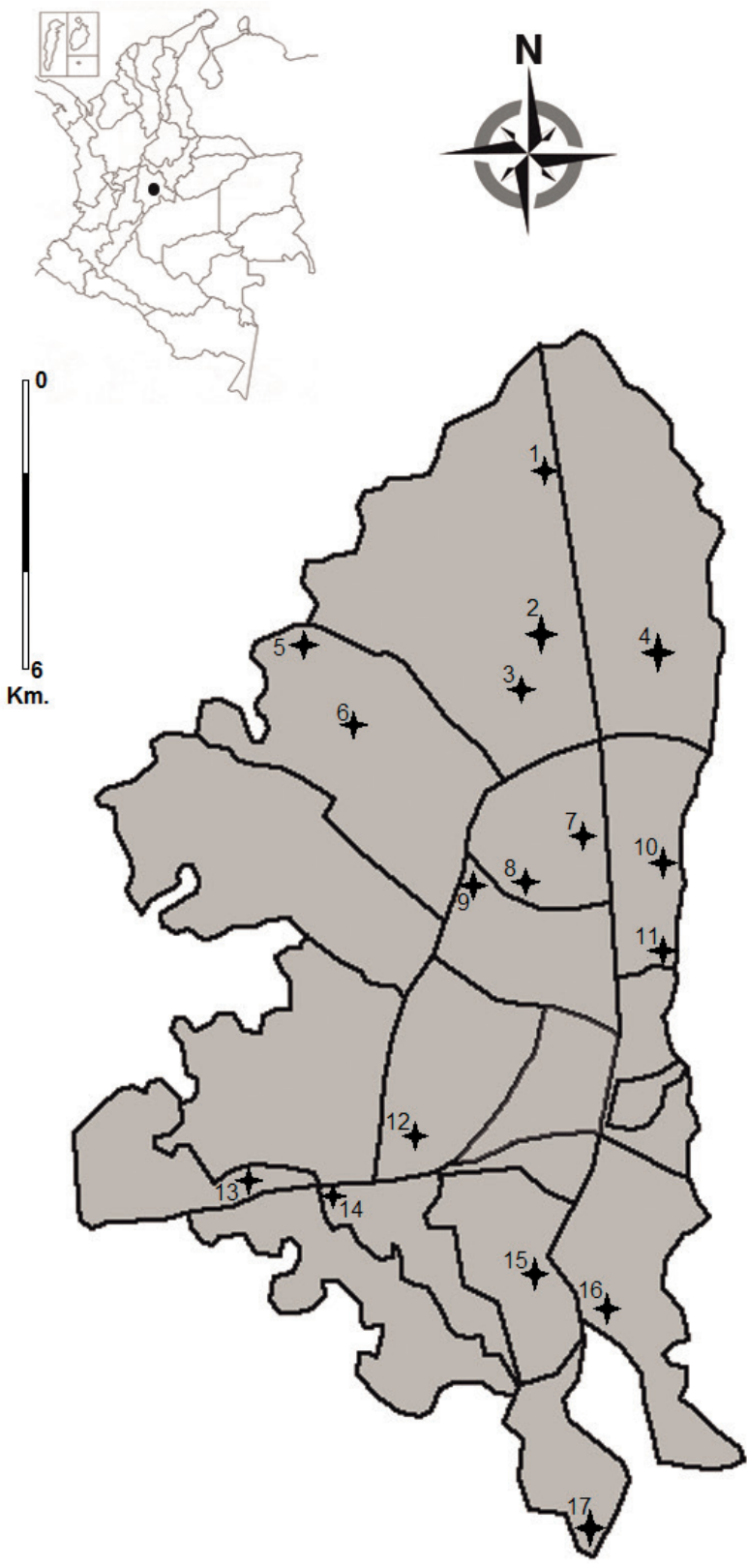

Fig. 1. Mapa político de Bogotá con los sitios muestreados por localidad en el pre-muestreo (A) y el muestreo cuantitativo (B). 1: Jardines del Recuerdo (Suba-A); 2: Niza (Suba-B); 3: Humedal Córdoba (Suba-A); 4: Lisboa, Los Cedros y Santa Bárbara (Usaquén-B); 5: Bolivia (Engativá-B); 6: Garcés Navas (Engativá-A); 7: Alcázares (Barrios Unidos-A y B); 8: Parque Los Novios (Barrios Unidos-A); 9: Parque Simón Bolívar (Teusaquillo-A); 10: Chicó Lago, Pardo Rubio y El Refugio (Chapinero-A y B); 11: campus Pontificia Universidad Javeriana y Parque Nacional Chapinero-A); 12: Alcalá (Puente Aranda-A y B); 13: Apogeo (Bosa-B); 14: Isla del Sol (Tunjuelito-A y B); 15: San José (Rafael Uribe Uribe-A y B); 16: 20 de Julio (San Cristóbal-B); 17: Ciudad de Usme (Usme-B). 
Tabla 1. Presencia (1) - ausencia (0) de especies de califóridos en 17 sitios del área urbana de Bogotá visitados durante el premuestreo.

\begin{tabular}{lccccccc}
\multicolumn{1}{c}{ Sitio } & R. roraima & C. nigribasis & C. verena & S. magellanica & L. sericata & C. vicina & C. albiceps \\
\hline 1. Jardines del Recuerdo & 0 & 0 & 1 & 1 & 1 & 1 & 0 \\
2. Niza & 0 & 0 & 0 & 1 & 1 & 1 & 0 \\
3. Humedal Córdoba & 0 & 0 & 0 & 0 & 0 & 0 & 0 \\
4. Lisboa & 0 & 0 & 0 & 0 & 1 & 1 & 0 \\
5. Bolivia & 0 & 0 & 1 & 1 & 1 & 1 & 0 \\
6. Garcés Navas & 0 & 0 & 0 & 0 & 1 & 0 & 0 \\
7. Alcázares & 0 & 0 & 1 & 0 & 1 & 1 & 0 \\
8. Parque Los Novios & 0 & 0 & 1 & 0 & 1 & 1 & 0 \\
9. Parque Bolívar & 0 & 0 & 0 & 0 & 0 & 0 & 0 \\
10. Chicó-Lago & 1 & 1 & 1 & 1 & 1 & 1 & 0 \\
11. Campus PUJ & 0 & 0 & 1 & 0 & 1 & 1 & 0 \\
12. Alcalá & 0 & 0 & 1 & 0 & 1 & 1 & 1 \\
13. Apogeo & 0 & 0 & 1 & 0 & 1 & 1 & 0 \\
14. Isla del Sol & 0 & 0 & 1 & 1 & 1 & 1 & 1 \\
15. San José & 0 & 0 & 1 & 1 & 1 & 1 & 0 \\
16. 20 de Julio & 0 & 0 & 0 & 1 & 1 & 1 & 0 \\
17. Ciudad de Usme & 0 & 0 & 0 & 1 & 1 & 1 & 0 \\
\hline
\end{tabular}

identificaron a especie utilizando las claves de McAlpine et al. (1981) y Amat et al. (2008). Se realizaron análisis de dominancia, diversidad y similitud utilizando los programas EstimateS 8.2 (Colwell 2011), BioDiversity Pro 2 y SPSS 19.

Análisis: El análisis estadístico se hizo con los datos del muestreo cuantitativo formal cuya representatividad se evaluó mediante una curva de acumulación de especies. Aunque el muestreo formal se llevó a cabo en días consecutivos pero diferentes según el sitio, este se hizo en condiciones climáticas similares. No obstante, se comparó la variación del esfuerzo de muestreo por sitio mediante una prueba de Kruskal Wallis para descartar la influencia de factores alineados, esto se hizo fijando el esfuerzo de muestreo por muestra como la diferencia entre el índice máximo de Shannon ( $\mathrm{H}^{\prime} \max$ ) y el índice de Shanon $\left(\mathrm{H}^{\prime}\right)$. Se estimó la riqueza y abundancia total de califóridos por sitio. La abundancia total de califóridos por sitio y la de las dos especies más abundantes se comparó utilizando pruebas de Kruskal Wallis, la de la tercera especie más abundante con una prueba de la mediana.
Las diferencias se analizaron con pruebas pareadas a posteriori de Dunn (para Kruskal Wallis) o tipo Tukey (para la prueba de la mediana). Los sitios en los cuales la especie analizada se ausento se consideraron significativamente diferentes de aquellos con registros de la especie y por lo tanto se excluyeron de las pruebas estadísticas.

\section{Resultados}

Durante el muestreo formal se colectaron seis especies: C. nigribasis, C. vicina, C. verena, $R$ roraima, $S$. magellanica y L. sericata. La composición encontrada durante el pre-muestreo fue diferente debido a la captura de Chrysomya albiceps (Wiedemann, 1819) en Alcalá e Isla del Sol y a variaciones locales en composición y abundancia de las demás especies (Tabla 1).

Durante el muestreo formal se colectaron 873 ejemplares (Tabla 2). La riqueza fluctuó entre 6 (ChicóLago) y 2 (Alcazares) especies por sitio, siendo 3 el valor más común (Tabla 2). De acuerdo con los estimadores de riqueza, la eficiencia del muestreo alcanzó entre 
Tabla 2. Abundancia total y relativa por sitio y por especie de moscas califóridas colectadas en 11 sitios de diferentes localidades de Bogotá. $\mathrm{N}=873 . \mathrm{S}=$ riqueza. $\mathrm{N}=$ abundancia. $\%=$ abundancia relativa.

\begin{tabular}{|c|c|c|c|c|c|c|c|c|c|c|c|c|c|}
\hline \multirow[b]{2}{*}{ Sitio } & \multirow[b]{2}{*}{$\mathrm{S}$} & \multicolumn{2}{|c|}{ R. roraima } & \multicolumn{2}{|c|}{ C. nigribasis } & \multicolumn{2}{|c|}{ C. verena } & \multicolumn{2}{|c|}{ S. magellanica } & \multicolumn{2}{|c|}{ L. sericata } & \multicolumn{2}{|c|}{ C. vicina } \\
\hline & & $\mathrm{n}$ & $\%$ & $\mathrm{n}$ & $\%$ & $\mathrm{n}$ & $\%$ & $\mathrm{n}$ & $\%$ & $\mathrm{n}$ & $\%$ & $\mathrm{n}$ & $\%$ \\
\hline Apogeo & 4 & 0 & 0 & 0 & 0 & 10 & 30 & 3 & 9 & 1 & 3 & 19 & 58 \\
\hline 20 de julio & 3 & 0 & 0 & 0 & 0 & 0 & 0 & 13 & 11 & 106 & 87 & 3 & 2 \\
\hline Ciudad de Usme & 3 & 0 & 0 & 0 & 0 & 0 & 0 & 21 & 49 & 3 & 7 & 19 & 44 \\
\hline Lisboa & 3 & 0 & 0 & 0 & 0 & 1 & 2 & 0 & 0 & 12 & 24 & 36 & 73 \\
\hline Chicó-Lago & 6 & 3 & 3 & 2 & 2 & 1 & 1 & 57 & 66 & 3 & 3 & 21 & 24 \\
\hline Alcalá & 3 & 0 & 0 & 0 & 0 & 0 & 0 & 1 & 3 & 8 & 21 & 29 & 76 \\
\hline San José & 3 & 0 & 0 & 0 & 0 & 0 & 0 & 4 & 5 & 59 & 69 & 22 & 26 \\
\hline Niza & 3 & 0 & 0 & 0 & 0 & 0 & 0 & 18 & 18 & 70 & 70 & 12 & 12 \\
\hline Alcázares & 2 & 0 & 0 & 0 & 0 & 0 & 0 & 0 & 0 & 103 & 58 & 76 & 42 \\
\hline Bolivia & 4 & 0 & 0 & 0 & 0 & 1 & 2 & 28 & 60 & 10 & 21 & 8 & 17 \\
\hline Isla del Sol & 3 & 0 & 0 & 0 & 0 & 0 & 0 & 1 & 1 & 76 & 83 & 13 & 16 \\
\hline Total & 6 & 3 & & 2 & & 13 & & 146 & & 441 & & 258 & \\
\hline
\end{tabular}

el 100\% (Chao 1 y ACE) y el 86\% (Jacknife 1) de lo observado (Figura 2), considerándose la muestra ampliamente representativa de los califóridos presentes en el área de estudio. El esfuerzo de muestreo por sitio no mostró diferencias significativas (Kruskal-Wallis, $\left.X^{2}=6,590 ; \mathrm{p}=0,763 ; \mathrm{n}=11\right)$.

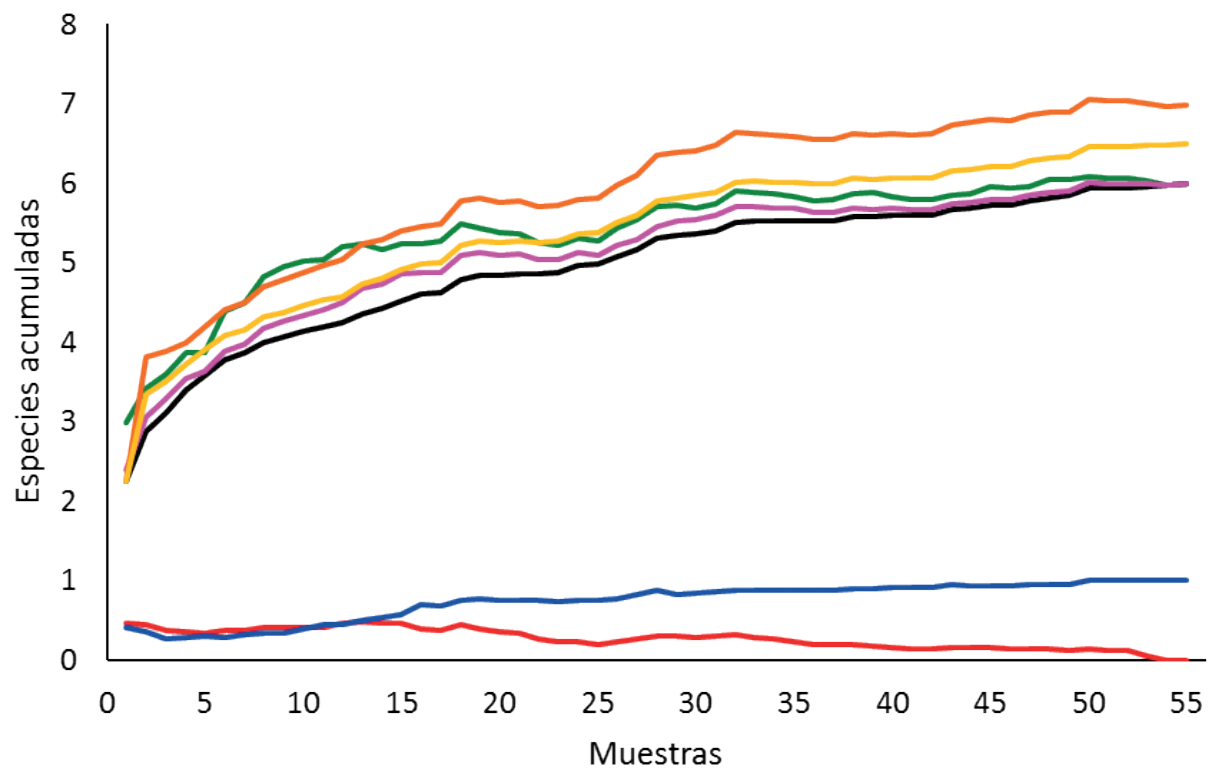

Fig. 2. Curva de acumulación de especies de moscas califóridas colectadas en 11 sitios de diferentes localidades de Bogotá. En negro: especies observadas acumuladas. En rojo: Singletons o especies colectadas con solo un individuo en toda la muestra. En azul: Doubletons, o especies colectadas con solo dos individuos en toda la muestra. En verde: estimador de riqueza de especies basado en la cobertura de la abundancia (ACE). En magenta: estimador de riqueza de especies de Chao para datos basados en abundancia (Chao 1). En naranja: estimador de riqueza de especies de jackknife de primer orden basado en la incidencia. En amarillo: estimador de riqueza de especies de bootstrap basado en la incidencia. 
La abundancia de califóridos total (todas las especies) no presentó diferencias significativas entre sitios $\left(X^{2}=14,862 ; p=0,137 ; n=11\right)$; sin embargo, la abundancia de cada especie fue variable. $L$. sericata fue la especie más abundante (451 individuos colectados), seguida de C. vicina (258 individuos; Tabla 2), ambas se capturaron en todos los sitios muestreados. Aunque que la abundancia de $L$. sericata varió significativamente entre sitios $\left(X^{2}=35,238 ; \mathrm{P}<0,001 ; \mathrm{n}=11\right)$, la de C. vicina no lo hizo $\left(X^{2}=10,820 ; \mathrm{p}=0.372 ; \mathrm{n}=11\right)$. La abundancia de L. sericata en 20 de julio, Alcázares, Niza, Isla del Sol y San José (los sitios de mayor abundancia), presentó diferencias significativas respecto de la observada en Lisboa, Chicó-Lago, Bolivia, Ciudad de Usme, Alcalá y Apogeo (según la prueba corregida de Dunn para rangos pareados, $\Delta \mathrm{ij}$ = 9,94; Supt. 1). Bolivia y Lisboa presentaron mayor abundancia que Apogeo, siendo significativamente diferentes de este, cuya abundancia promedio fue la menor $\left(\chi_{\text {Apogeo }}=0,2 ;\right.$ Tipo HSD de Tukey; $q_{0.05, \infty, 11}$ $=9,9447 ; \mathrm{p}<0,05 ; \mathrm{n}=10$ Tabla 2). La abundancia de S. magellanica también varió significativamente entre sitios $\left(X^{2}=25,714 ; \mathrm{p}<0,001 ; \mathrm{n}=11\right)$, siendo significativamente diferentes Niza y Chicó-Lago, y Pardo Rubio y El Refugio de Alcalá e Isla del Sol, que fueron los sitios con menor abundancia promedio (Tipo HSD de Tukey; $\mathrm{q}_{0.05, \infty, 9}=4,387 ; \mathrm{P}<0,05 ; \mathrm{n}$ $=10)$.

La diversidad $\left(\mathrm{H}^{\prime}\right)$ fue mayor en Bolivia, seguida de Apogeo, Chicó-Lago y Ciudad de Usme y menor en 20 de Julio. 20 de Julio, al tiempo, presentó la mayor dominancia (D), seguida de Isla del Sol y Alcalá; Apogeo, Bolivia y Ciudad Usme presentaron la menor dominancia (Tabla 3).

El análisis de similitud utilizando el índice cualitativo de Jaccard agrupó en una unidad a Isla del Sol, Niza, San José, Alcalá, Ciudad de Usme y 20 de Julio (Figura 3). En estos sitios L. sericata, C. vicina y $S$. magellanica formaron un ensamble de especies que puede ser característico del suroriente de Bogotá para la época del estudio. Niza fue el único sitio ubicado al norte de la ciudad que compartió este ensamble (Figura 1). Apogeo y Bolivia fueron asociadas por la presencia de L. sericata, C. vicina, S. magellanica y $C$. verena; un grupo que podría ser otro ensamble que caracterizaría al borde oriental de la ciudad. Lisboa compartió con los dos grupos anteriores a L. sericata, C. vicina y C. verena. Chicó-Lago, el sitio más rico, y Alcázares, el más pobre, presentaron la menor similitud $(33,33 \%)$.

Tabla 3. Índices de diversidad de Shannon (H'), uniformidad ( $\mathrm{H}^{\prime} / \mathrm{H}^{\prime}$ max $)$ y dominancia de Simpson (D) de moscas califóridas colectadas en 11 sitios de diferentes localidades de Bogotá.

\begin{tabular}{|c|c|c|c|}
\hline Sitio & Shannon $\left(\mathrm{H}^{\prime}\right)$ & $H^{\prime} / H^{\prime} \max$ & Simpson (D) \\
\hline Bolivia & 1,021 & 0,736 & 0,417 \\
\hline Apogeo & 1,004 & 0,724 & 0,415 \\
\hline Chicó-Lago & 0,99 & 0,552 & 0,485 \\
\hline Ciudad de Usme & 0,897 & 0,816 & 0,425 \\
\hline Niza & 0,813 & 0,740 & 0,532 \\
\hline San José & 0,747 & 0,680 & 0,546 \\
\hline Alcázares & 0,682 & 0,984 & 0,509 \\
\hline Lisboa & 0,65 & 0,591 & 0,592 \\
\hline Alcalá & 0,63 & 0,573 & 0,617 \\
\hline Isla del Sol & 0,509 & 0,463 & 0,703 \\
\hline 20 de Julio & 0,452 & 0,411 & 0,765 \\
\hline
\end{tabular}


$\mathrm{Al}$ incluir en el análisis de similitud las densidades por especie y sitio (índice de Bray-Curtis) Isla del Sol y San José fueron los lugares más similares $(88,48 \%$ de similitud según índice de Bray-Curtis). Estos se asociaron a Niza y 20 de Julio (Figura 3) debido principalmente a la alta densidad de L. sericata y C. vicina (Tabla 2). 20 de Julio y Apogeo presentaron la menor similitud cuantitativa $(9,03 \%$ de similitud de Bray-Curtis; Figura 3), debido a su composición y a la mayor abundancia de C. vicina y C. verena (Tabla 2). Las asociaciones generadas por Bray-Curtis muestran dos grandes agrupamientos que pueden asociarse a dos ambientes urbanos contrastantes: áreas de la ciudad con zonas verdes amplias y áreas con zonas verdes reducidas o ausentes. Las zonas verdes amplias o su cercanía son característicos del primer grupo de sitios asociados: Alcalá, Lisboa, Chicó-Lago, Bolivia, Ciudad de Usme y Apogeo (Figura 3); mientras que el segundo grupo, Niza, Isla del Sol, Alcázares y 20 de Julio, se caracteriza por estar en localidades con áreas verdes de menor tamaño.
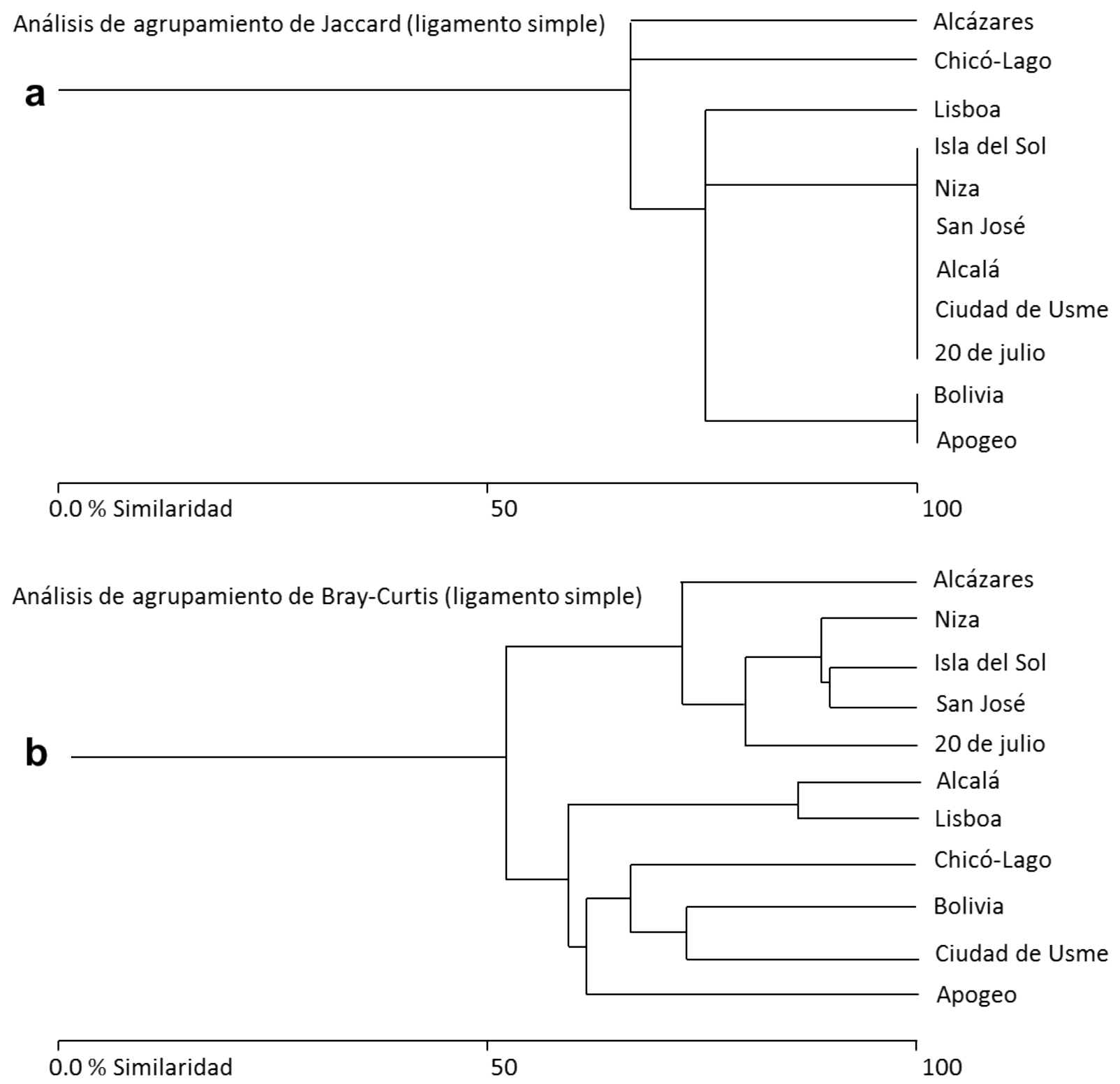

Fig. 3. Análisis de agrupamiento simple según índices de similitud cualitativo de Jaccard (a) y cuantitativo de Bray-Curtis (b) de moscas califóridas colectadas en 11 sitios de diferentes localidades de Bogotá. 


\section{Discusión}

Si bien el uso de cadáveres es el mejor método para detectar fauna forense, genera un fuerte impacto por los olores y afectación visual que produce sobre los vecinos de la localidad, en especial en áreas densamente pobladas. Este problema ha obligado a realizar la mayoría de estudios experimentales de entomología forense en zonas rurales, semirurales o periféricas. Ejemplo de esto son los trabajos de Segura et al. (2009) y Camacho (2005) realizados en zonas cercanas al área urbana de Bogotá. Ante este problema, el usó del jameo ad hoc direccionado constituye un método alternativo para detectar fauna forense en áreas urbanas densamente habitadas. La versatilidad del método fue manifiesta en este estudio; ni Camacho (2005), ni Segura et al. (2009), capturaron a $R$ roraima y Segura et al. (2009) no capturaron las dos especies urbanas más frecuentes (C. vicina y $L$ sericata). Adicionalmente, la eficiencia del jameo ad hoc direccionado se puede inferir de la comparación de la presente muestra, 873 ejemplares y siete especies entre pre-muestreo y muestreo, con lo obtenido por Pinilla-Beltrán et al. (2012), quienes colectaron seis especies de califóridos en 3951 especímenes utilizando trampas tipo Ferreira y jameo con cebo. No obstante, es evidente que la colecta directa de especímenes sobre cadáveres humanos es el montaje ideal (Barreto et al. 2002).

Teniendo en cuenta que utilizamos un método alternativo al uso de cadáveres, fue claro que la abundancia específica de califóridos varió significativamente entre sitios, modificando variables locales como la riqueza, diversidad y dominancia, aunque la abundancia total por sitio hubiese sido homogénea. Estos cambios fueron evidentes en los análisis de agrupamiento de Bray-Curtis y Jaccard. Las variaciones se deben principalmente a la ausencia o presencia de $C$. verena y $S$. magellanica, más la variación en la abundancia de $L$. sericatay $C$. vicina, siempre presentes (Tabla 2). C. verena y S. magellanica fueron registradas como especies con preferencias rurales por Amat (2009) y Pinilla-Beltrán et al. (2012). Sin embargo, en términos generales, la composición difiere de la esperada de acuerdo con Amat (2009). Varias especies registradas en Bogotá Amat (2009), como Cocbliomyia macellaria (Fabricius, 1775), Compsomyiops melloi (Dear 1985), Paralucilia fulvinota (Bigot, 1877) o Chlorobracbycoma splendida (Townsend, 1918) no fueron colectadas. Dicho autor reportó a C. macellaria en la UPZ Santa Cecilia
(Engativá), un sitio cercano a Bolivia (Engativá); C. melloi y $P$. fulvinota no tienen indicaciones más específicas y C. splendida en humedal la conejera (Amat 2009), área cercana a Niza y Bolivia. Al respecto, Pinilla-Beltrán et al. (2012) tampoco registraron dichas especies, ni a C. albiceps, registrada en el pre-muestreo. Estas diferencias entre los tres estudios evidencian la variabilidad de la fauna local de califóridos, donde algunas especies como $C$. nigribasis y $R$. roraima se restringen a sitios muy específicos, mientras que otras como C. albiceps, C. macellaria, C. melloi, C. splendida o P. fulvinota pueden presentar una marcada estacionalidad.

Al comparar la diversidad y dominancia entre sitios, se evidencia una posible relación entre la disponibilidad de vegetación y la diversidad local de califóridos. Ambientes urbanos cercanos a zonas rurales con vegetación arbórea o áreas con mayor frecuencia de parques, rondas de ríos y zonas verdes presentaron mayor valor de diversidad y baja dominancia (Tabla 2); ejemplo de ello fueron Bolivia (Engativá), Apogeo (Bosa), Chico-Lago y Ciudad de Usme (Usme). Las características de la vegetación en los sitios de muestreo parecen modificar variables como la diversidad y dominancia. Esto sugiere que el tamaño de las zonas verdes puede ser determinante en la distribución de califóridos, lo cual tiene sentido dado el carácter nectarívoro de los califóridos adultos (Marshall 2012) y puede ser un aspecto importante a tener en cuenta al momento de analizar las especies asociadas a un cuerpo.

Los ambientes creados por el hombre afectan significativamente la composición de califóridos, pues en áreas urbanas se registra mayor abundancia en muchas especies de muscoideos (Figueroa-Roa \& Linhares 2002). Mientras L. sericata y C. vicina, ambas consideradas urbanas (Pinilla-Beltrán et al. 2012), fueron las especies más abundantes y definieron los agrupamientos de Bray-Curtis, la presencia de especies reportadas como asinantrópicas (C. nigribasis y $R$ roraima) definieron las asociaciones de similitud cualitativa (Jaccard) (PinillaBeltrán et al. 2012; Figura 3). La ausencia de estas especies coincidió con un incremento en la densidad de $L$. sericata, C. vicina y S. magellanica, que si bien se encontraron distribuidas en toda el área urbana de Bogotá, fueron mucho más abundantes en el suroriente, donde la densidad de áreas verdes es menor. Estas especies podrían formar un ensamble característico del suroriente de Bogotá, al menos para la época del estudio. 
Las observaciones resaltan la importancia de los estudios de variación local como herramienta para deducir casos de traslado de cadáveres y como herramienta de apoyo a medios sucesionales de estimación del IPM.

La ciudad no es homogénea y cuenta con muchas zonas verdes de diferentes tamaños (SDA 2007), las cuales presentan más espacios donde se podrían ocultar larvas de califóridos en fase de pre-pupación, ya que estos insectos generalmente se entierran en dicha fase (Byrd \& Castner 2001). Los ambientes cubiertos por asfalto y concreto presentarían menos espacios para ocultarse, por lo cual las especies desarrollarían tolerancias distintas a estas exigencias del ambiente urbano. La cercanía de zonas verdes y el tamaño de las mismas puede ser la razón principal por la cual se presentan variaciones en la composición y abundancia de califóridos entre ambientes urbanos.

L. sericata, la especie más abundante, ha sido registrada en Usaquén (Segura et al. 2009), Santa Fe y Chapinero (Pinilla-Beltrán et al. 2012); las localidades de Bosa, San Cristóbal, Usme, Puente Aranda, Rafael Uribe Uribe, Suba, Barrios Unidos, Engativá y Tunjuelito representan registros nuevos en el área urbana e indican una distribución generalizada (Tabla 2). En Bogotá fue mucho más abundante hacia el centro, en áreas muy urbanizadas (Figura 4F).
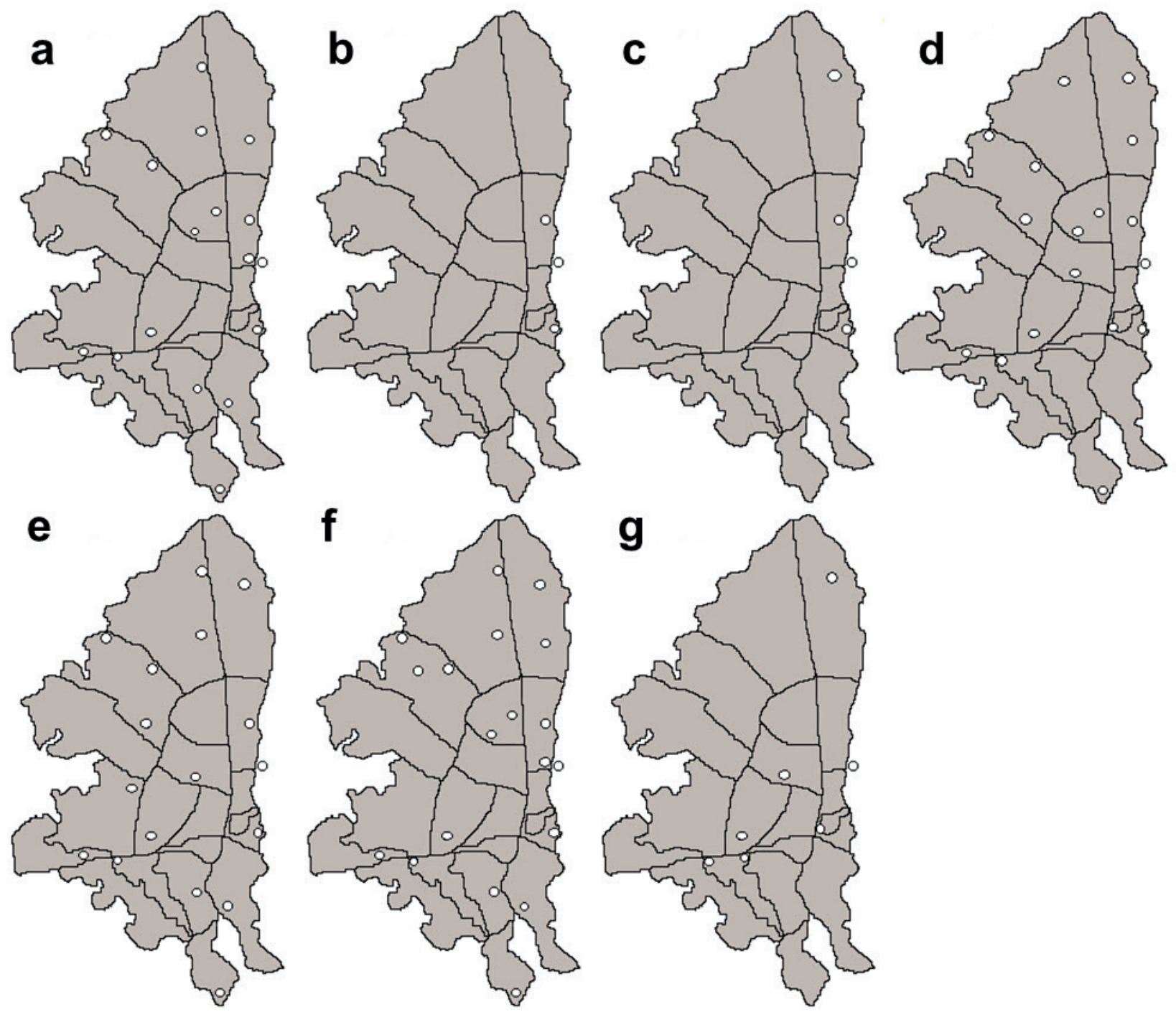

Fig. 4. Reportes históricos de Calliphora vicina (a), Roraimomusca roraima (b), Calliphora nigribasis (c), Compsomyiops verena (d), Sarconesiopsis magellanica (e), Lucilia sericata (f) y Chrysomya albiceps (g), para el área urbana de Bogotá. La información corresponde a lo encontrado en este estudio y la bibliografía existente. 
Los registros obtenidos complementan lo encontrado por Pinilla-Beltrán et al. (2012), Barrios \& Wolf (2011) y Segura et al. $(2009,2011)$ en estudios realizados en zonas rurales cercanas. C. vicina se distribuyó homogéneamente en todas las regiones muestreadas en la ciudad (Figura 4). C. vicina se ha reportado en las localidades de Usaquén (Segura et al. 2009), Santa Fe, Chapinero y Engativá (Pinilla-Beltrán et al. 2012); gracias a este estudio, a sus registros urbanos se suma: Bosa, San Cristóbal, Usme, Puente Aranda, Rafael Uribe Uribe, Suba, Barrios Unidos, y Tunjuelito (Tabla 2, Figura 4A).

La abundancia de L. sericata, C. verena, S. magellanica, C. nigribasis y R. roraima, presentó diferencias significativas entre sitios. $R$ roraima ya se había reportado por primera vez para Bogotá en Chapinero y Santa Fe (PinillaBeltrán et al. 2012). Se confirma su presencia en Bogotá, en áreas más urbanizadas (Chicó-Lago), pero cercanas a amplia cobertura arbórea que caracteriza los cerros orientales (SDA 2007). R. roraima se distribuye en el centro-oriente de Bogotá, en ambientes cercanos a los cerros orientales (Figura 4B). C. nigribasis se reporta para Usaquén, Santa Fe y Chapinero (Pinilla-Beltrán et al. 2012, Segura et al. 2009), distribuyéndose en áreas menos intervenidas al nororiente y centro-oriente de Bogotá (Figura 4C).

C. verena se ha reportado en Usaquén (Segura et al. 2009), Santa Fe, Chapinero, Engativá (Pinilla-Beltrán et al. 2012), Teusaquillo, Suba (Amat 2009), y con este estudio en Tunjuelito, Barrios Unidos, Puente Aranda y Bosa. Su máxima abundancia se reportó en Apogeo, sitio en donde, en contraste, la abundancia de $L$. sericata fue significativamente menor (Tabla 2, Figura 4D). C. verena podría distribuirse por toda el área urbana de Bogotá, pero su frecuencia seria mayor en temporada seca, en especial en sitios con áreas verdes mayores y con heces humanas depositadas al aire libre, en concordancia con las observaciones de Amat (2009). S. magellanica se distribuye casi uniformemente en la ciudad (Figura 4E), se ha reportado en Usaquén (Segura et al. 2009), Santa Fe, Engativá, Chapinero (Pinilla-Beltrán et al. 2012), Teusaquillo y Kennedy (Amat 2009); en este trabajo se amplían dichos registros a Bosa, San Cristóbal, Usme, Puente Aranda, Rafael Uribe Uribe y Tunjuelito (Tabla 2, Figura 4E).

C. albiceps, colectada en Puente Aranda (Alcalá) y Tunjuelito (Isla del Sol) durante el pre-muestreo, se ha colectado en Usaquén (Segura et al. 2009), Teusaquillo,
Chapinero y Santa Fe (Amat 2009). Esta especie se distribuiría principalmente al sur de la ciudad en ciertas épocas del año (Figura 4), pues no fue registrada por Pinilla-Beltrán et al. (2012), quienes muestrearon por mucho más tiempo. Algunos estudios señalan a C. albiceps como urbana (Moretti \& Godoy 2013; Montoya et al. 2009), se dice que supera en abundancia a C. vicina en dichos ambientes, sin embargo estos trabajos se realizaron en zonas relativamente menos urbanizadas que las del presente trabajo. En los ambientes relativamente mas urbanizados C. albiceps se asocia más a ambientes semejantes a paisajes rurales (Bonacci et al. 2011, Aballay et al. 2012, Battán-Horenstein et al. 2012, De Souza \& Zuben 2012, Lambiase \& Camerini 2011,Prado e Castro et al. 2011), evento observado en este trabajo. Sin embargo, para entender completamente la distribución de estas especies en el área urbana haría falta un programa de monitoreo estacional multianual con sitios de muestreo definidos.

\section{Conclusión}

L. sericata, C. vicina, más otra especie, posiblemente $S$. magellanica sea la composición de califóridos más frecuente en Bogotá; estas especies se distribuyen por toda su área urbana en la época de lluvias. L. sericata, C. vicina y $S$. magellanica, las especies más abundantes y ampliamente distribuidas, conformarían un ensamblaje característico del suroriente de Bogotá, donde la densidad de áreas verdes es menor. L. sericata fue la especie dominante en zonas céntricas y altamente urbanizadas. La distribución más localizada de $\mathrm{R}$ roraima y $C$. nigribasis, se puede asociar a zonas con vegetación arbórea por lo cual estarían restringidas a áreas cercanas a los cerros orientales. Las variaciones en riqueza, composición y abundancia que se evidencian aquí pueden servir como apoyo para la identificación de casos de traslado de cuerpos y en menor medida para la predicción de variaciones locales de la sucesión cadavérica en Bogotá. Se necesita un estudio de mayor duración para identificar posibles variaciones por sitio y por estacionalidad en especies de califóridos.

\section{Agradecimientos}

A los miembros del laboratorio de entomología de la Pontificia Universidad Javeriana por su constante apoyo y asesoría. Agradecimiento por soporte financiero a Colciencias Proyecto P-2011-00168; convocatoria 521, Vicerrectoría de investigaciones de la Pontificia Universidad Javeriana. 


\section{Conflictos de intereses}

Los autores manifestamos que no tenemos intereses diferentes a los intereses científicos asociados a los resultados de este trabajo.

\section{Referencias}

Aballay FH, Murua AF, Acosta JC, Centeno ND (2012) Succession of carrion fauna in the arid region of San Juan Province, Argentina and its forensic relevance. Neotropical Entomology 41(1):27-31 doi: 10.1007/s13744-011-0005-9

Amat E (2009) Contribución al conocimiento de las Chrysomyinae y Toxotarsinae (Diptera: Calliphoridae) de Colombia. Revista Mexicana de Biodiversidad 80:693-708

Amat E, Vélez MC, Wolff M (2008) Clave ilustrada para la identificación de los géneros y las especies de califóridos (Diptera: Calliphoridae) de Colombia. Caldasia 30(1):231244

Amendt J, Richards CS, Campobasso CP, Zehner R, Hall MJR (2010) Forensic Entomology: applications and limitations. Forensic Science, Medicine and Pathology 7(4):379392 doi: 10.1007/s12024-010-9209-2

Anderson GS (2000) Minimum and maximum development rates of some forensically important Callophoridae (Diptera). Journal of Forensic Science 45(4):824-832

Barreto M, Burbano ME, Barreto P (2002) Flies (Calliphoridae, Muscidae) and beetles (Silphidae) from human cadavers in Cali, Colombia. Mem Inst Oswaldo Cruz Rio de Janeiro 97(1):137-138

Barrios M, Wolff M (2011) Initial study of arthropods succession and pig carrion decomposition in two freshwater ecosystems in the Colombian Andes. Forensic Science International 212(1-3):164-172 doi: 10.1016/j. forsciint.2011.06.008. Epub 2011 Jul 7

Battán-Horenstein M, Rosso B, Dolores-García M (2012) Seasonal structure and dynamics of sarcosaprophagous fauna on pig carrion in a rural area of Cordoba (Argentina): Their importance in forensic science. Forensic Science International 217:146-156 doi: 10.1016/j. forsciint.2011.10.043

Bonacci T, Zetto-Brandmayr T, Brandmayr P, Vercillo V, Porcelli F (2011) Successional patterns of the insect fauna on a pig carcass in southern Italy and the role of Crematogaster scutellaris (Hymenoptera: Formicidae) as a carrion invader. Entomological Science 14:125-132 doi: 10.1111/j.1479-8298.2010.00423.x

Brundage A, Bros S, Honda JY (2011) Seasonal and habitat abundance and distribution of some forensically important blow flies (Diptera: Calliphoridae) in Central California. Forensic Science International 212(1-3):115120 doi: 10.1016/j.forsciint.2011.05.023
Byrd H, Castner JL (2001) Forensic entomology: the utility of arthropods in legal investigations. CRC Press LLC, USA

Camacho G (2005) Sucesión de la entomofauna cadavérica y ciclo vital de Calliphora vicina (Diptera: Calliphoridae) como primera especie colonizadora, utilizando cerdo blanco (sus scrofa) en Bogotá. Revista Colombiana de Entomología 31(2):189-197

Colwell, R. K. 2011. EstimateS, Version 8.2: Statistical estimation of species richness and shared species from samples. Consultado el 30 de mayo 2013. http://viceroy. eeb.uconn.edu/Colwell/

DANE, Departamento Nacional de Estadísticas (2005) Boletín Censo General 2005, Perfil Bogotá Bogotá (2005) DANE. www.dane.gov.C. Consultado el 30 de mayo 2013 http://www.dane.gov.co/files/censo2005/PERFIL_ PDF_CG2005/11001T7T000.PDF

De Souza CR, Zuben CJV (2012) Diversity and synanthropy of Calliphoridae (Diptera) in the Region of Rio Claro, SP, Brazil. Neotropical Entomology 41(3):243-248 doi: 10.1007/ s13744-012-0037-9

Figueroa-Roa L, Linhares A (2002) Sinantropía de los Calliphoridae (Diptera) de Valdívia, Chile. Neotropical Entomology 31(2):233-239

Fremdt H, Amendt J (2013) Species composition of forensically important blow flies (Diptera: Calliphoridae) and flesh flies (Diptera: Sarcophagidae) through space and time. Forensic Science International, In Press, Accepted Manuscript, Available online 19 December 2013 DOI: http://dx.doi.org/doi:10.1016/j.forsciint.2013.12.010

IDEAM, Instituto de Hidrología, Meteorología y Estudios Ambientales (2007) Estudio de la caracterización climática de Bogotá y cuenca alta del rio Tunjuelo. IDEAM. Bogotá, D.C., Colombia

Lambiase S, Camerini G (2011) Spread and habitat selection of Chrysomya albiceps (Wiedemann) (Diptera: Calliphoridae) in northern Italy: forensic implications. Journal of Forensic Science 57(3):799-801 doi:10.1111/j.15564029.2011.02027.x

Macana NL (2012) Comportamiento del suicidio en Colombia, 2011. Forensis 13(1):245-265

Mariluis JC, Schnack JA, Mulieri PP, Patitucci LD (2008) Calliphoridae (Diptera) from wild, suburban, and urban sites at three Southeast Patagonian localities. Revista de la Sociedad Entomológica Argentina 67(1-2):107-114

Marshall, SA (2012) Flies: the natural history and diversity of Diptera. Firefly Books. Ontario, Canada

McAlpine JF, Peterson BV, Shewell GE, Teskey HJ, Vockeroth JR, Wood DM (1981) Manual of Neartic Diptera. Minister of Supply and Services Canada. Ottawa, Canadá 
Montoya AL, Sánchez JD, Wolff M (2009) Sinantropía de Calliphoridae (Diptera) del municipio La Pintada, Antioquía-Colombia. Revista Colombiana de Entomología 35(1):73-82

Moretti TC, Godoy WAC (2013) Spatio-temporal dynamics and preference for type of bait in necrophagous insects, particularly native and introduced blow flies (Diptera: Calliphoridae). Journal of Medical Entomology 50(2):415-424

Pinilla-Beltrán YT, Segura NA, Bello FJ (2012) Synanthropy of Calliphoridae and Sarcophagidae (Diptera) in Bogotá, Colombia. Neotropical Entomology 41(3):237-242 doi: 10.1007/s13744-012-0036-x

Prado e Castro C, Sousa JP, Arnaldos MI, Gaspar J, García MD (2011) Blowflies (Diptera: Calliphoridae) activity in sun exposed and shaded carrion in Portugal. Annales de la Societe Entomologique de France 47(1-2):128-139

Prinkkilä ML, Hanski I (1995) Complex competitive interactions in four species of Lucilia blowflies. Ecological Entomology 20(3):261-272

\section{Cambios en composición y abundancia de califóridos de} interés forense en Bogotá

Resumen. Los califóridos son los primeros artrópodos en colonizar cadáveres y varían localmente en composición y abundancia; siendo utilizados para estimar el intervalo postmortem e inferir traslado de cadáveres. Reconocer variaciones locales en composición y abundancia es importante para identificar eventos de traslado. Se estudió la variación en composición y abundancia de califóridos en 11 sitios de Bogotá. Se realizó un pre-muestreo (estación seca) para escoger método de captura e identificar los sitios de muestreo y un muestreo formal (estación lluviosa) usando jameo ad hoc direccionado. Se comparó la riqueza, diversidad, dominancia y composición por sitio. Se registraron seis especies: Lucilia sericata, Roraimomusca roraima, Compsomyiops verena, Calliphora nigribasis, Calliphora vicina y Sarconesiopsis magellanica. Mayor riqueza se evidenció en Chicó-Lago (Chapinero), y menor en Alcázares (Barrios Unidos). Bolivia (Engativá) fue el más diverso y 20 de julio (San Cristóbal) el menos diverso, la dominancia fue mayor en 20 de julio y menor en Apogeo (Bosa). La composición y abundancia por especie variaron entre sitios lo cual puede ser evidencia de apoyo para casos de traslado de cuerpos en Bogotá.

Palabras clave: Califóridos; composición y abundancia; traslado de cadáveres; Bogotá.
Ricaurte AI (2012) Comportamiento del homicidio. Colombia 2011. Forensis 13(1):67-99

SDA, Secretaría Distrital de Ambiente (2007) Atlas Ambiental de Bogotá D.C. Secretaría Distrital de Ambiente, Alcaldía Mayor de Bogotá, D.C. Bogotá, Colombia

Segura NA, Bonilla MA, Usaquén W, Bello F (2011) Entomofauna resource distribution associated with pig cadavers in Bogotá. Medical and Veterinary Entomology 25:46-52 doi: 10.1111/j.1365-2915.2010.00933.x

Segura NA, Usaquén W, Sánchez MC, Chuaire L, Bello F (2009) Succession pattern of cadaverous entomofauna in a semi-rural area of Bogotá, Colombia. Forensic Science International187:66-72 doi: 10.1016/j.forsciint.2009.02.018

Villareal H, Alvarez M, Córdoba S, Escobar F, Fagua G, et al. (2006) Manual de métodos para el desarrollo de inventarios de Biodiversidad. Instituto de Investigación de Recursos Biológicos Alexander von Humboldt. Programa Inventarios de Biodiversidad. Grupo de Exploración y Monitoreo Ambiental. Bogotá, Colombia

Alteraçóes em composiçáo e abundância de califorídeos de interesse forense em Bogotá

Resumo. Os califorídeos são os primeiros artrópodes em colonizar cadáveres e variam localmente em composição e abundância; sendo utilizados para estimar o intervalo post-mortem e inferir transferência de corpos. Reconhecer variações locais em composição e abundância é importante para identificar acontecimentos de transferência. Estudou-se a variação na composição e abundância de califorídeos em 11 locais de Bogotá. Realizou-se uma amostragem previa (estação seca) para escolher o método de captura e identificar os sítios de amostragem e realizar uma amostragem formal (estação chuvosa) utilizando jameo ad hoc direccionado. Comparou-se a riqueza, diversidade, dominância e composição por localidade. Registaram-se seis espécies: Lucilia sericata, Roraimomusca roraima, Compsomyiops verena, Calliphora nigribasis, Calliphora vicina y Sarconesiopsis magellanica. Evidenciou-se maior riqueza em Chicó-Lago (Chapinero), e menor em Alcázares (Barrios Unidos). Bolivia (Engativá) foi o mais diverso e 20 de julio (San Cristóbal) o menos diverso, a dominância foi maior em 20 de julio e menor em Apogeo (Bosa). A composição e abundância por espécie variaram entre os locais, o qual pode ser evidencia de apoio para casos de transferência de corpos em Bogotá.

Palavras-chave: Califorídeos; composição e abundancia; transferência de corpos; Bogotá. 